Theatre Research in Canada

Recherches théâtrales au Canada

\title{
Hommage à Joyce Doolittle
}

\section{Hélène Beauchamp}

Volume 41, numéro 2, 2020

Special Issue on Performance For/By/With Young People in Canada Numéro spécial sur la performance pour/par/avec les jeune dans un contexte canadien

URI : https://id.erudit.org/iderudit/1074787ar

DOI : https://doi.org/10.3138/tric.41.2.02.fr

Aller au sommaire du numéro

Éditeur(s)

University of Toronto Press

ISSN

1196-1198 (imprimé)

1913-9101 (numérique)

Découvrir la revue

Citer ce document

Beauchamp, H. (2020). Hommage à Joyce Doolittle. Theatre Research in Canada / Recherches théâtrales au Canada, 41(2), 198-200.

https://doi.org/10.3138/tric.41.2.02.fr d'utilisation que vous pouvez consulter en ligne.

https://apropos.erudit.org/fr/usagers/politique-dutilisation/ 


\section{Hommage à Joyce Doolittle}

L'artiste, militante et chercheure foyce Doolittle, née le 5 avril 1928, est décédée le 6 mars 2020, alors que nous terminions la préparation de ce numéro thématique. Elle a été l'une des premières chercheures du Canada anglais à s'intéresser dans ses écrits aux jeunes personnes et aux artistes qui créent du théâtre pour/ parlavec les jeunes.

Dans A Mirror of Our Dreams: Children and the Theatre in Canada (1979) de foyce Doolittle et Zina Barnieh - toujours le seul ouvrage consacré entièrement au théâtre jeunesse au Canada —, on retrouve un chapitre sur le théâtre au Québec rédigépar Hélène Beauchamp dans lequel il est écrit : "Il est temps de réévaluer et d'élargir notre façon de concevoir l'enfance... [et] les arts sont essentiels à ce processus, et le théâtre fournit des outils puissants et uniques pour ce travail critique » (24, traduction).

2uarante et un ans plus tard, cet appel à l'action est tout aussi pertinent.

Foyce Doolittle et Hélène Beauchamp ont fait ouvve de pionnières dans la recherche menée au Canada sur les jeunes personnes et la performance. En préparant ce numéro, nous marchons avec bumilité dans leurs pas. Nous sommes très touchées que Beauchamp ait accepté d'écrire cet hommage à sa collègue.

-Rédactrices invitées, Sandra Chamberlain-Snider et Heather Fitzsimmons Frey

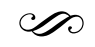

HÉLĖNE BEAUCHAMP

Université du Québec à Montréal, Montréal, Québec, Canada

Joyce aimait la vie. Elle aimait la vie chez les personnes qui l'entouraient, et le cercle de ses amitiés était grand. Elle était passionnée de théâtre et a cherché, tout au long de sa vie, à ce que le plus grand nombre de personnes y aient accès. C'est ainsi que, très tôt, elle a vu le potentiel créateur que recelait le théâtre produit pour et avec les enfants et les jeunes.

C'est vers 1976 que Joyce prend contact avec moi alors que je commence, de mon côté, à mesurer l'ampleur du mouvement qui allait donner lieu à la grande aventure de création dramatique et théâtrale du « nouveau » théâtre pour l'enfance et la jeunesse. Ce mouvement, artistique, social et politique était à l'œuvre en Europe depuis le début des années i960. Les artistes québécois et canadiens étaient à l'affût de ces réalisations qui exigeaient des compétences spécifiques, une grande capacité d'adaptation au risque, et l'obligation d'inventer de nouvelles structures. Ce serait un théâtre différent à tous les points de vue. Quel bonheur! Je trouvais en Joyce une collègue précieuse.

Nous allions, pour notre plus grand plaisir et dans le partage amical, constituer les deux pôles canadiens - d'est en ouest - de Vancouver à Moncton en passant par Calgary et Montréal de la reconnaissance de plein droit de cet " autre " théâtre (voir la figure I). Nous allions faire valoir les liens essentiels entre les jeunes et les artistes, entre le théâtre et les pratiques éducatives.

Joyce m'a lancé un premier défi : participer à $A$ Mirror of Our Dreams: Children and the Theatre in Canada (1979), publication qui en officialisait l'existence. Reconnaissant le principe des solidarités internationales, Joyce s'inscrit aussi dans le réseau de l'Association internationale du 


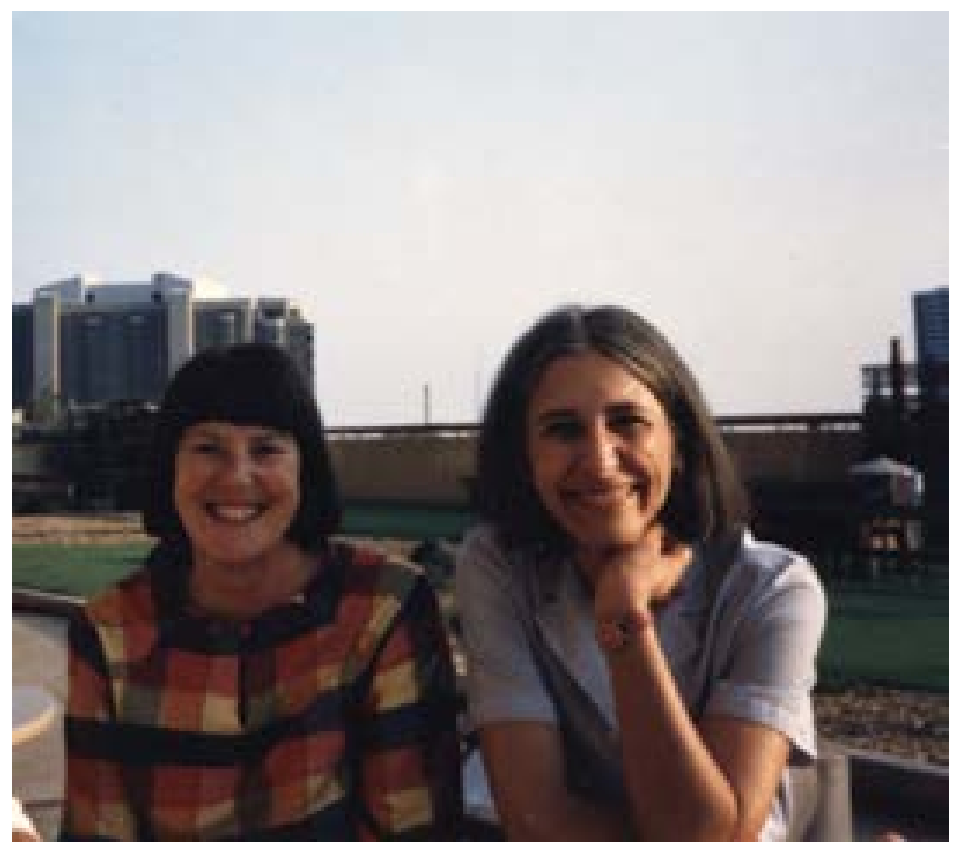

Figure 1. Joyce

Doolittle et Hélène

Beauchamp,

International Drama

Education Research

Symposium à Toronto, mai 1989.

théâtre pour l'enfance et la jeunesse (ASSITEJ), créée en I965. Au cours de plusieurs voyages, elle nous représente en Russie et s'assure de la fondation d'ASSITEJ-Canada. Suivant la piste ainsi ouverte, je suis conférencière invitée au Congrès mondial d'ASSITEJ à Lyon en I98I.

Joyce me lance un second défi : concevoir un numéro de la revue d'ASSITEJ consacré entièrement au théâtre québécois et canadien pour les jeunes publics (voir la figure 2). Publié en 1985, Le Défi du théâtre pour enfants au Québec et au Canada-The Challenge of Children's Theatre in 2uebec and in Canada, comprend des articles écrits en anglais et en français, par des artistes de premier rang dont : Suzanne Lebeau, Dennis Foon, Brigitte Haentjens, Graham Whitehead, Maureen Labonté.

Joyce a été une collègue exceptionnelle, généreuse, énergique, d'une créativité et d'un humour uniques. Nous nous sommes rencontrées souvent, lors des colloques de l'Association canadienne de la recherche théâtrale/Canadian Association for Theatre Research, et tout par ticulièrement à Calgary, en I994, où j'ai prononcé son hommage autour d'un BBQ dont les steaks étaient de poids!

Et alors que mes cheveux blanchissaient lentement mais sûrement, elle tenait à me rappeler, souriant sous ses cheveux noirs : « You know, I don't dye my hair. » Comme si je pouvais l'oublier!

Avec toute mon amitié. Hélène

\section{Bibliographie}

Beauchamp, Hélène, dir., Le Défi du théâtre pour enfants au 2uébec et au Canada-The Challenge of Children's Theatre in 2uebec and in Canada, numéro spécial de la revue, Théâtre Enfance feunesse, vol. I-2, ASSITEJ - International, 1985.

Doolittle, Joyce et Zina Barnieh, avec Hélène Beauchamp. A Mirror of Our Dreams: Children and the Theatre in Canada. Vancouver, Talonbooks, 1979. 


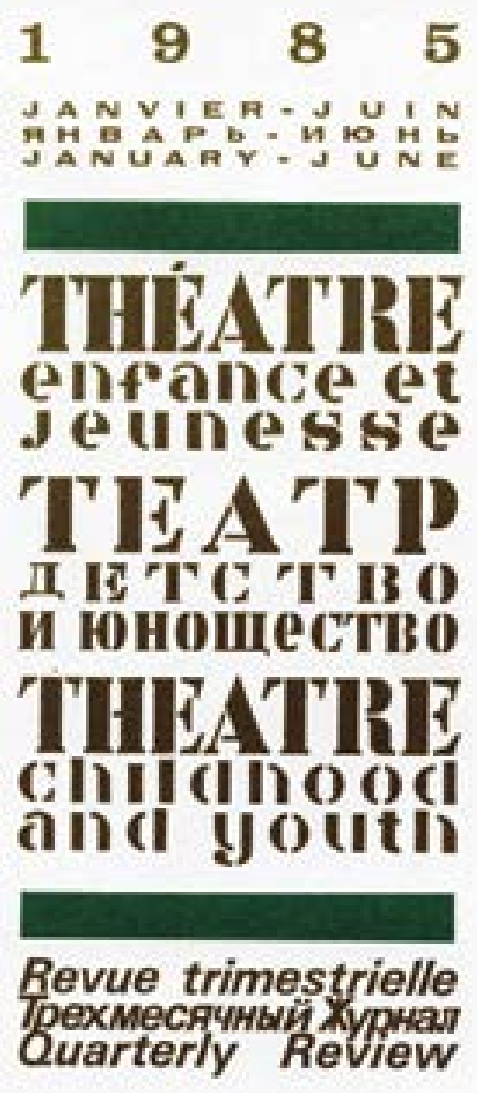

Figure 2. La couverture du numéro spécial du Théâtre enfance jeunesse paru en 1985.

\section{Contributrice}

HÉLĖNE BEAUCHAMP a enseigné à l'Université d'Ottawa de 1966 à I975 et ensuite à l'École supérieure de théâtre de l'Université du Québec à Montréal jusqu'en 2003. Elle a publié une longue série d'ouvrages fondamentaux sur l'histoire du théâtre, la pédagogie artistique, le théâtre pour l'enfance et la jeunesse et sur les théâtres professionnels du Canada francophone. En 2009 , l'Association canadienne pour la recherche théâtrale lui attribue le Prix de Carrière et l'UQAM, le statut de professeure émérite; en mai 2015, elle devient membre honoraire de la Société québécoise d'études théâtrales. 